\title{
The blow up analysis of solutions of the elliptic sinh-Gordon equation
}

\author{
Jürgen Jost • Guofang Wang • \\ Dong Ye · Chunqin Zhou
}

Received: 5 September 2006 / Accepted: 18 May 2007 / Published online: 13 July 2007

(C) Springer-Verlag 2007

\begin{abstract}
In this paper, using a geometric method we show that the blow-up values of the elliptic sinh-Gordon equation are multiples of $8 \pi$.
\end{abstract}

Mathematics Subject Classification (2000) 35 J60 - 58E20

\section{Introduction}

The aim of this paper is to explore the relationship between the analytic aspects of the elliptic sinh-Gordon equation

$$
u_{z \bar{z}}+\lambda \sinh u=0
$$

in particular the blow-up analysis, on a two-dimensional surface $(\Sigma, g)$ and its geometric interpretation in terms of constant mean curvature surfaces and harmonic maps. We therefore hope that our work will provide a better understanding of solutions of the sinh-Gordon

The fourth named author supported partially by NSFC of China (No. 10301020).

J. Jost (凶)

Max Planck Institute for Mathematics in the Sciences, Inselstr. 22, 04013 Leipzig, Germany

e-mail: jjost@mis.mpg.de

G. Wang

Faculty of Mathematics, University Magdeburg, 39016 Magdeburg, Germany

e-mail: gwang@math.uni-magdeburg.de

D. Ye

Département de Mathématiques, UMR 8088, Université de Cergy-Pontoise, BP 222,

95302 Cergy-Pontoise, France

e-mail: dong.ye@u-cergy.fr

C. Zhou

Department of Mathematics, Shanghai Jiaotong University, Shanghai 200240, China

e-mail: cqzhou@sjtu.edu.cn 
equation. In fact, this equation plays a very important role in the study of the construction of constant mean curvature surfaces initiated by Wente. See [41] or the next section.

Wente's work [40] on the existence problem for constant mean curvature surfaces and the simultaneous work of Sacks-Uhlenbeck [31] on two-dimensional harmonic map started the investigation of blow-up phenomena for variational problems that possess a noncompact invariance group and represent limiting cases where the Palais-Smale condition just fails. Wente's seminal work then lead to the subsequent work of Steffen [35], Struwe [36] and Brezis-Coron [5] which completed the understanding of the blow-up for constant mean curvature surfaces from a geometric point of view (the analogous analysis for harmonic maps was achieved by Brezis-Coron [6] and Jost [14].)

Spruck [34] introduced an analytic point of view into the study of the sinh-Gordon equation (1). When $\Sigma$ is a rectangle in $\mathbb{R}^{2}$, he studied the behavior of nonnegative solutions of (1) with a Dirichlet boundary condition as $\lambda$ tends to zero. In particular, he proved that a sequence of nonnegative and nontrivial solutions $\left(\lambda_{k}, u_{k}\right)$ for the Dirichlet problem of (1) tends to the Green function $-2 \log |G(z)|^{2}$ as $\lambda_{k} \rightarrow 0$ in a suitable sense. Here $G(z)$ is the conformal map of $\Sigma$ onto the unit disk.

Equation (1) arises also from many mathematical and physical problems. See for instance $[9,18,21,23-25,28,42]$, and the references therein. In this paper, with the help of differential geometry, we shall investigate the blow up analysis of solutions to (1) when $\Sigma$ is a Riemann surface or a bounded smooth domain in $\mathbb{R}^{2}$, and we shall give a more precise asymptotic behavior when the sequence of solutions blows up as $\lambda_{n} \rightarrow \lambda$. Let $v_{n}$ be a sequence of solutions of (1), i.e. $v_{n}$ satisfies

$$
-\Delta v_{n}=\lambda_{n}\left(e^{v_{n}}-e^{-v_{n}}\right) \quad \text { in } \Sigma
$$

with the condition

$$
\int_{\Sigma} \lambda_{n}\left(e^{v_{n}}+e^{-v_{n}}\right) d v_{g} \leq C<\infty
$$

and $\lim _{n \rightarrow \infty} \lambda_{n}=\lambda$. In order to state our main result, we define the blow-up set of the sequence $\left\{v_{n}\right\}$ by

$$
\begin{aligned}
& S_{1}=\left\{x \in \Sigma \mid \exists x_{n} \rightarrow x \text { such that } v_{n}\left(x_{n}\right) \rightarrow \infty\right\}, \\
& S_{2}=\left\{x \in \Sigma \mid \exists x_{n} \rightarrow x \text { such that }-v_{n}\left(x_{n}\right) \rightarrow \infty\right\} .
\end{aligned}
$$

Up to subsequences, it is not difficult to show (see Lemma 3.2) that $S_{1}$ and $S_{2}$ are finite sets. For $p \in S_{1} \cup S_{2}$, set

$$
m_{1}(p)=\lim _{r \rightarrow 0} \lim _{n \rightarrow \infty} \int_{B_{r}(p)} \lambda_{n} e^{v_{n}} d v_{g} \text { and } m_{2}(p)=\lim _{r \rightarrow 0} \lim _{n \rightarrow \infty} \int_{B_{r}(p)} \lambda_{n} e^{-v_{n}} d v_{g} .
$$

These are two different types of blow-up. Our main theorem is

Theorem 1.1 The blow-up values $m_{1}$ and $m_{2}$ are multiples of $8 \pi$.

This is an analogue of the result of Li and Shafrir [19] for the Liouville equation

$$
-\Delta u=\lambda e^{u} .
$$

Analytically, the blow-up analysis of the Liouville equation can be seen as a special case $\left(S_{2}=\varnothing\right)$ of that for the sinh-Gordon equation. 
In view of a relationship established in [25],

$$
\left[m_{1}(p)-m_{2}(p)\right]^{2}=8 \pi\left[m_{1}(p)+m_{2}(p)\right],
$$

a direct consequence of Theorem 1.1 is

Corollary 1.2 The blow-up values of the sinh-Gordon equation (2) can only be

$$
\left(m_{1}(p), m_{2}(p)\right)=8 \pi\left(\frac{\ell(\ell-1)}{2}, \frac{\ell(\ell+1)}{2}\right) \text { or } 8 \pi\left(\frac{\ell(\ell+1)}{2}, \frac{\ell(\ell-1)}{2}\right)
$$

for some integer $\ell>0$.

This result was in fact conjectured in [25].

The proof of Theorem 1.1 is more geometric. We shall use differential geometry of surfaces of constant mean curvature to transfer our problem into a blow-up phenomenon for harmonic maps. Then we apply a result about no loss of energy during bubbling off for a sequence of harmonic maps, which was proved in [15,26]. See also [11] and [20]. A direct analytic proof of Theorem 1.1 is an interesting problem. However, it seems to be subtle, at least the method presented in [19] seems difficult to generalize to the sinh-Gordon equation. There are many examples in partial differential equations in which the geometric structure of equations plays a crucial role. Good examples are Wente's inequality [39] and Helein's proof [12] of the regularity of weak harmonic maps. See also the recent work [30]. For the blow-up analysis of the Toda system see $[16,17]$.

Problem 1 Is the constant $\ell$ in Corollary 1.2 one?

Without any boundary constraint, we believe that there are examples with $\ell>1$. However it is not easy to give an example (cf. [8] for the Liouville equation). We shall consider this problem elsewhere. With a suitable boundary condition, for examples that $\left(\max _{\partial \Sigma} v_{n}-\min _{\partial \Sigma} v_{n}\right)$ is uniformly bounded, we believe that $\ell=1$.

\section{The sinh-Gordon equation and constant mean curvature surfaces}

Let $f: \Sigma \rightarrow \mathbb{R}^{3}$ be a conformal immersion of a surface in $\mathbb{R}^{3}$. Its first fundamental form is

$$
I=e^{2 u}|d z|^{2}
$$

and the second fundamental form is

$$
I I=\frac{Q}{2} d z^{2}+H e^{2 u} d z d \bar{z}+\frac{\bar{Q}}{2} d \bar{z}^{2} .
$$

Here $H$ is the mean curvature and $Q d z^{2}$ is the Hopf differential. The Gauss-Codazzi equation gives

$$
-\Delta u=H^{2} e^{2 u}-|Q|^{2} e^{-2 u} \text { and } Q_{\bar{z}}=e^{2 u} H_{z},
$$

where $Q_{\bar{z}}=\partial Q / \partial \bar{z}$ and $H_{z}=\partial H / \partial z$. When this conformal immersion has constant mean curvature (denoted by CMC), i.e. $H=$ constant, then the Hopf differential $Q$ is holomorphic. When $Q=0$ (for instance in the case where $\Sigma$ is a sphere), equation (7) gives the Liouville equation (after a rescaling)

$$
-\Delta u=e^{2 u}
$$


When $Q=c \neq 0$ (for instance in the case where $\Sigma$ is a torus), we have (after a rescaling and transformation as $u \mapsto u+\sigma$ ) the sinh-Gordon equation

$$
-\Delta u=e^{2 u}-e^{-2 u} .
$$

Hence from a CMC surface, we have a solution of the sinh-Gordon equation (9). Vice versa, from a solution of (9), one can get a CMC surface if $\Sigma$ is a simply connected domain. This plays a very important role in the construction of constant mean curvature immersions, which was initiated by Wente (see also [1,3,27]).

Let $v$ be the unit outer normal vector of the immersion $f$. One can check that $v$, as a map from $\Sigma \rightarrow \mathbb{S}^{2}$, the Gauss map, satisfies

$$
-\Delta v=2 H_{\bar{z}} f_{z}+2 Q_{\bar{z}} e^{-2 u} f_{\bar{z}}+\left(H^{2} e^{2 u}+|Q|^{2} e^{-2 u}\right) v .
$$

Thus $f$ has constant mean curvature if and only if $-\Delta v$ is a multiple of $v$, which is equivalent to $v$ being a harmonic map from $\Sigma \rightarrow \mathbb{S}^{2}$, a well known result, see e.g. [4] or Lemma 4.2 below. In this case, we have

$$
|\nabla v|^{2}=H^{2} e^{2 u}+|Q|^{2} e^{-2 u}
$$

Hence the condition (3) is equivalent to the Gauss map having finite energy. Therefore as mentioned, the blow-up analysis of the sinh-Gordon equation becomes equivalent to the blow-up analysis of CMC surfaces.

\section{Preliminary results}

We assume first that $\Sigma=\Omega$ is a smooth bounded domain in $\mathbb{R}^{2}$. We consider the blow-up analysis of the following equation

$$
\begin{cases}-\Delta v_{n}=\lambda_{n}\left(e^{v_{n}}-e^{-v_{n}}\right) & \text { in } \Omega \\ v_{n}=0 & \text { on } \partial \Omega\end{cases}
$$

under a finite energy condition

$$
\int_{\Omega} \lambda_{n}\left(e^{v_{n}}+e^{-v_{n}}\right) d x \leq C<\infty .
$$

We have then

Lemma 3.1 Let $v_{n}$ be a family of $C^{2}$ solutions of (12) verifying (13). For $B_{r}(x) \subset \Omega$, if we have

$$
\lim _{n \rightarrow \infty} \int_{B_{r}(x)} \lambda_{n} e^{v_{n}} d x<8 \pi \quad\left(\text { resp. } \lim _{n \rightarrow \infty} \int_{B_{r}(x)} \lambda_{n} e^{-v_{n}} d x<8 \pi\right),
$$

then there is a constant $C>0$ such that

$$
\max _{B_{r / 2}(x)} \lambda_{n} e^{v_{n}} \leq C \quad\left(\text { resp. } \max _{B_{r / 2}(x)} \lambda_{n} e^{-v_{n}} \leq C\right) .
$$


Proof We use the following inequality in [32]: If $p$ is a positive $C^{2}$ solution satisfying $-\Delta \log p \leq p$ in $B_{r}\left(x_{0}\right) \subset \mathbb{R}^{2}$, then

$$
\log p\left(x_{0}\right) \leq \frac{1}{\pi r^{2}} \int_{B_{r}\left(x_{0}\right)} \log p(y) d y-2 \log \left(1-\frac{1}{8 \pi} \int_{B_{r}\left(x_{0}\right)} p(y) d y\right)_{+} .
$$

By our assumption,

$$
\int_{B_{r}(x)} \lambda_{n} e^{v_{n}} d x \leq C_{0}<8 \pi
$$

for sufficiently large $n$. Taking $p=\lambda_{n} e^{v_{n}}$, we get $-\Delta \log p \leq p$ in $\Omega$, so that for $n$ large enough and any $z \in B_{r / 2}(x)$,

$$
v_{n}(z) \leq \frac{4}{\pi r^{2}} \int_{B_{r / 2}(z)} v_{n}(y) d y-2 \log \left(1-\frac{C_{0}}{8 \pi}\right) .
$$

Moreover, by elliptic theory, $v_{n}$ is uniformly bounded in $W_{0}^{1, q}(\Omega)$ for any $q<2$. The above inequality means that $v_{n}$ is uniformly upper bounded in $B_{r / 2}(x)$. We can do the same for $\lambda_{n} e^{-v_{n}}$.

Set $e\left(v_{n}\right)=\lambda_{n}\left(e^{v_{n}}+e^{v_{n}}\right)$ and

$$
S=\left\{x \in \Omega \mid \lim _{\delta \rightarrow 0}\left[\limsup _{n \rightarrow \infty} \int_{B_{\delta}(x)} e\left(v_{n}\right) d x\right] \geq 8 \pi\right\} .
$$

Lemma 3.2 We have $S=S_{1} \cup S_{2}$ and $v_{n}$ is uniformly bounded in any compact $K \subset \Omega \backslash S$.

From this Lemma, it is easy to see that $S$ consists of a finite number of points. Another immediate consequence of Lemma 3.2 is

Proposition 3.3 Let $v_{n}$ be a sequence of solutions to (12) satisfying $\lim _{n \rightarrow \infty}\left\|v_{n}\right\|_{\infty}=\infty$ and $\lim _{n \rightarrow \infty} \lambda_{n}=0$. Up to a subsequence, there exists a finite, non empty set $S=S_{1} \cup S_{2}$ in $\Omega$ such that

$$
\lambda_{n} e^{v_{n}} d x \rightarrow \sum_{x_{0} \in S_{1}} m_{1}\left(x_{0}\right) \delta_{x_{0}} \text { and } \lambda_{n} e^{-v_{n}} d x \rightarrow \sum_{y_{0} \in S_{2}} m_{2}\left(y_{0}\right) \delta_{y_{0}}
$$

Moreover, $v_{n}$ converges to $G$ in $C_{l o c}^{\infty}(\Omega \backslash S)$ and in $W_{0}^{1, q}(\Omega)$ for any $q<2$. Here, $G$ is the Green function defined by

$$
\begin{cases}-\Delta G=\sum_{p \in S}\left[m_{1}(p)-m_{2}(p)\right] \delta_{p} & \text { in } \Omega \\ G=0 & \text { on } \partial \Omega\end{cases}
$$

where $m_{i}(p) \geq 8 \pi$ if $p \in S_{i}$ and we define $m_{i}(p)=0$ if $p \in S \backslash S_{i}(i=1,2)$.

When $\lim _{n \rightarrow \infty} \lambda_{n}>0$, we get the asymptotic behavior of $v_{n}$ as follows.

Proposition 3.4 Let $v_{n}$ be a sequence of solutions to (12) verifying (13) with $\lim _{n \rightarrow \infty} \lambda_{n}=$ $\lambda>0$. Up to a subsequence, we have

- either $v_{n}$ is bounded in $L_{\mathrm{loc}}^{\infty}(\Omega)$; 
- or there exists a non empty set $S \subset \Omega$ such that $S_{1}=S_{2}=S=\left\{p_{1}, \ldots, p_{k}\right\}$ and $v_{n}$ is bounded in $L_{\text {loc }}^{\infty}(\Omega \backslash S)$. Moreover, in the sense of measures,

$$
\lambda_{n} e^{v_{n}} d x \rightarrow r_{1}(x) d x+\sum_{1 \leq j \leq k} m_{1}\left(p_{j}\right) \delta_{p_{j}}, \quad \lambda_{n} e^{-v_{n}} d x \rightarrow r_{2}(x) d x+\sum_{1 \leq j \leq k} m_{2}\left(p_{j}\right) \delta_{p_{j}}
$$

where $r_{i} \in L^{1}(\Omega) \cap C_{l o c}^{\infty}(\Omega \backslash S)$ and $m_{1}\left(p_{j}\right), m_{2}\left(p_{j}\right) \geq 8 \pi$.

Proof The dichotomy result is an immediate consequence of Theorem 4.2 in [22] with $u_{1, n}=v_{n}$ and $u_{2, n}=-v_{n}$. We need only to remark that it is impossible to have $u_{i, n} \rightarrow-\infty$ uniformly on any open set of $\Omega$, since if it is the case, the condition in (13) is false by $u_{1, n}+u_{2, n} \equiv 0$ in $\Omega$.

Problem 2 Does the second case of Proposition 3.4 really occur?

Next we want to characterize the blow-up value at blow up points in $\Omega$ for solutions of (2), which is essentially achieved in [25] by using a symmetrization method. Here we follow the arguments of [7] and use the Pohozaev identity.

Lemma 3.5 With the notations in Proposition 3.3 or 3.4, for any $p \in S$, we have

$$
\left[m_{1}(p)-m_{2}(p)\right]^{2}=8 \pi\left[m_{1}(p)+m_{2}(p)\right] .
$$

Proof Without loss of generality, we assume that $p=0$ and for sufficiently small $r_{0}>0$, $B_{r_{0}}(0) \cap S=\{0\}$. Multiplying $x \cdot \nabla v_{n}$ to (12) and integrating in $B=B_{r}(0)$ with $r \in\left(0, r_{0}\right)$, we get the following Pohozaev identity

$$
\begin{aligned}
& r \int_{\partial B_{r}}\left(\frac{\partial v_{n}}{\partial v}\right)^{2} d \sigma-\frac{r}{2} \int_{\partial B_{r}}\left|\nabla v_{n}\right|^{2} d \sigma \\
& \quad=2 \int_{B_{r}} \lambda_{n}\left(e^{v_{n}}+e^{-v_{n}}\right) d x-r \int_{\partial B_{r}} \lambda_{n}\left(e^{v_{n}}+e^{-v_{n}}\right) d \sigma .
\end{aligned}
$$

Since in both cases $\lambda=0$ or $\lambda>0$, we have always

$$
v_{n}(x) \rightarrow-\frac{m_{1}(0)-m_{2}(0)}{2 \pi} \log |x|+H(x) \quad \text { in } C_{\mathrm{loc}}^{\infty}(B \backslash\{0\})
$$

with some $H \in C^{\infty}(B \backslash\{0\})$, letting first $n \rightarrow \infty$, then $r \rightarrow 0$, we obtain

$$
\left[m_{1}(0)-m_{2}(0)\right]^{2}=8 \pi\left[m_{1}(0)+m_{2}(0)\right] .
$$

The proof is completed.

From our analysis, we can see that it is not necessary to have the same coefficients in front of $e^{v_{n}}$ and $e^{-v_{n}}$, and we can also combine the study of the cases $\lambda>0$ and $\lambda=0$. Therefore similar results hold, for example, for the following equation

$$
\begin{cases}-\Delta v_{n}=\frac{\lambda_{1} e^{v_{n}}}{\int_{\Omega} e^{v_{n}} d x}-\frac{\lambda_{2} e^{-v_{n}}}{\int_{\Omega} e^{-v_{n}} d x} & \text { in } \Omega \\ v_{n}=0 & \text { on } \partial \Omega .\end{cases}
$$

We leave the details for interested readers. 
Consider now the equations (12) or (15) on a surface $\Sigma$ with boundary, instead of a bounded domain $\Omega \subset \mathbb{R}^{2}$. Using local isothermal charts, we can remark that by changing everywhere $8 \pi$ to a suitable positive constant $\varepsilon_{0}$, all the arguments for Lemmas 3.1, 3.2 continue to work, so we still have a finite set of singularities $S$. Using again a local analysis, we can repeat the proof of Propositions 3.3, 3.4 and Lemma 3.5 by just replacing the Green function $G$ by the one of $\Sigma$. See for example [25].

By the same argument, similar results hold for solutions of

$$
-\Delta v_{n}=\frac{\lambda e^{v_{n}}}{\int_{\Sigma} e^{v_{n}} d x}-\frac{\lambda e^{-v_{n}}}{\int_{\Sigma} e^{-v_{n}} d x} \text { in } \Sigma \text { and } \int_{\Sigma} v_{n} d x=0,
$$

with a closed surface $\Sigma$.

\section{Proof of the main theorem}

In this section we prove the main theorem, Theorem 1.1. First we follow closely the paper of Bobenko [4] to construct a harmonic map from a solution of a sinh-Gordon equation. Let $\Omega$ be a simply connected domain in $\mathbb{R}^{2}$ and let $u$ be a solution of

$$
-\Delta u=2 \lambda_{1} e^{u}-2 \lambda_{2} e^{-u} \quad \text { in } \Omega,
$$

for two positive constants $\lambda_{1}$ and $\lambda_{2}$. Define two matrices

$$
\mathcal{U}=\frac{1}{2}\left(\begin{array}{cc}
u_{z} & -\sqrt{\lambda_{2}} e^{-\frac{u}{2}} \\
\sqrt{\lambda_{1}} e^{\frac{u}{2}} & 0
\end{array}\right), \quad \mathcal{V}=\frac{1}{2}\left(\begin{array}{cc}
0 & -\sqrt{\lambda_{1}} e^{\frac{u}{2}} \\
\sqrt{\lambda_{2}} e^{-\frac{u}{2}} & u_{\bar{z}}
\end{array}\right) .
$$

It is easy to check the following equivalence.

Lemma 4.1 $u$ is a solution of (17) if and only if $\mathcal{U}$ and $\mathcal{V}$ satisfy

$$
\mathcal{U}_{\bar{z}}-\mathcal{V}_{z}+[\mathcal{U}, \mathcal{V}]=0
$$

Let $\mathbb{H}$ denote the algebra of quaternions and $\{\mathbf{1}, \mathbf{i}, \mathbf{j}, \mathbf{k}\}$ be the standard basis of $\mathbb{H}$ with $\mathbf{i j}=\mathbf{k}, \mathbf{j k}=\mathbf{i}$ and $\mathbf{k i}=\mathbf{j}$. It is convenvient to express quaternions using matrices. In order to do so, we only need to identify the Pauli matrices as follows

$$
\begin{array}{ll}
\sigma_{1}=\left(\begin{array}{cc}
0 & 1 \\
1 & 0
\end{array}\right)=i \mathbf{i}, & \sigma_{2}=\left(\begin{array}{cc}
0 & -i \\
i & 0
\end{array}\right)=i \mathbf{j}, \\
\sigma_{3}=\left(\begin{array}{cc}
1 & 0 \\
0 & -1
\end{array}\right)=i \mathbf{k}, \quad\left(\begin{array}{ll}
1 & 0 \\
0 & 1
\end{array}\right)=\mathbf{1} .
\end{array}
$$

We can also identify $\mathbb{R}^{3}$ with the space of imaginary quaternions $\operatorname{Im} \mathbb{H}$ by

$$
A=-i \sum_{i=1}^{3} a_{i} \sigma_{i} \in \operatorname{Im} \mathbb{H} \Longleftrightarrow A=\left(a_{1}, a_{2}, a_{3}\right) \in \mathbb{R}^{3} .
$$

Let $\mathbb{H}_{*}=\mathbb{H} \backslash\{0\}$ be the multiplicative quaternion group. Any element $\phi$ in $\mathbb{H}_{*}$ can be expressed by

$$
\phi=\left(\begin{array}{cc}
a & b \\
-\bar{b} & \bar{a}
\end{array}\right) \quad \text { with }|a|^{2}+|b|^{2} \neq 0 .
$$


Define a one-form $\alpha$ valued in the algebra $\mathbb{H}$ by

$$
\alpha=\mathcal{U} d z+\mathcal{V} d \bar{z} .
$$

We also point out that $d+\alpha$ is a flat connection if and only if $\mathcal{U}, \mathcal{V}$ satisfy (19).

Consider the following equation

$$
d \phi \cdot \phi^{-1}=\alpha,
$$

for $\phi: \Omega \rightarrow \mathbb{H}_{*}$. Equation (20) is equivalent to

$$
\phi_{z}=\mathcal{U} \phi \text { and } \phi_{\bar{z}}=\mathcal{V} \phi .
$$

The compatibility condition for (21) is just (19), which is equivalent to (17) by Lemma 4.1. Therefore for any solution $u$ of (17), by the Frobenius theorem, on a simply connected domain $\Omega$, equation (20) has a solution $\phi: \Omega \rightarrow \mathbb{H}_{*}$. Let $x_{0} \in \Omega$ be a fixed point. With the following normalization

$$
\phi\left(x_{0}\right)=e^{u(0) / 4} \mathbf{1}
$$

the solution of (21) is unique. First we claim that

$$
\operatorname{det} \phi=e^{u / 2} \text {. }
$$

Note that $\operatorname{det} \phi \neq 0$ and $\operatorname{set} h=\log (\operatorname{det} \phi)$. In view of (21) it is easy to verify that

$$
\begin{aligned}
& h_{z}=\operatorname{tr}\left(\phi^{-1} \phi_{z}\right)=\operatorname{tr}(\mathcal{U})=u_{z} / 2, \\
& h_{\bar{z}}=\operatorname{tr}\left(\phi^{-1} \phi_{\bar{z}}\right)=\operatorname{tr}(\mathcal{V})=u_{\bar{z}} / 2 .
\end{aligned}
$$

Hence $h-\frac{u}{2}$ is a constant over $\Omega$. By the normalization (22), we have $\operatorname{det} \phi=e^{u / 2}$.

From the map $\phi$, we define a map $N: B_{1} \rightarrow \mathbb{S}^{2}$ by

$$
N=\phi^{-1} \mathbf{k} \phi \text {. }
$$

It is clear that

$$
\phi^{-1}=\frac{1}{\operatorname{det} \phi}\left(\begin{array}{cc}
\bar{a} & -b \\
\bar{b} & a
\end{array}\right)
$$

and

$$
N=\frac{-i}{\operatorname{det} \phi}\left(\begin{array}{cc}
|a|^{2}-|b|^{2} & 2 \bar{a} b \\
2 a \bar{b} & |b|^{2}-|a|^{2}
\end{array}\right) .
$$

It follows that $\|N\|^{2}=1$ and $N: \Omega \rightarrow \mathbb{R}^{3}=\operatorname{Im} \mathbb{H}$. It is easy now to check that

Lemma 4.2 The map $N$ satisfies the harmonic map equation

$$
-\Delta N=|\nabla N|^{2} N \text {. }
$$

Proof In fact, $N$ is the Gauss map of a CMC surface and hence is a harmonic map. This is a well-known fact, see for instance [4]. Here for convenience of readers, we give a direct computation. We set

$$
F_{z}=-i e^{u / 2} \phi^{-1}\left(\begin{array}{ll}
0 & 0 \\
1 & 0
\end{array}\right) \phi, \quad F_{\bar{z}}=-i e^{u / 2} \phi^{-1}\left(\begin{array}{ll}
0 & 1 \\
0 & 0
\end{array}\right) \phi .
$$

By [4], $F: \Omega \rightarrow \mathbb{R}^{3}=\operatorname{Im} \mathbb{H}$ is also well-defined and is a CMC surface, but here we will not discuss the surface $F$. 
As $\left(\phi^{-1}\right)_{z}=-\phi^{-1} \mathcal{U}$ and $\left(\phi^{-1}\right)_{\bar{z}}=-\phi^{-1} \mathcal{V}$, a direct computation gives

$$
F_{\bar{z} z}=F_{z \bar{z}}=\frac{\sqrt{\lambda_{1}} e^{u}}{2} N
$$

and

$$
F_{z z}=u_{z} F_{z}+\frac{\sqrt{\lambda_{2}}}{2} N, \quad F_{\bar{z} \bar{z}}=u_{\bar{z}} F_{\bar{z}}+\frac{\sqrt{\lambda_{2}}}{2} N .
$$

We get also

$$
N_{z}=-\phi^{-1} \mathcal{U} \mathbf{k} \phi+\phi^{-1} \mathbf{k} \mathcal{U} \phi=\phi^{-1}[\mathbf{k}, \mathcal{U}] \phi=-\sqrt{\lambda_{1}} F_{z}-\sqrt{\lambda_{2}} e^{-u} F_{\bar{z}}
$$

and $N_{\bar{z}}=\phi^{-1}[\mathbf{k}, \mathcal{V}] \phi=-\sqrt{\lambda_{2}} e^{-u} F_{z}-\sqrt{\lambda_{1}} F_{\bar{z}}$. Since $\left\|F_{z}\right\|^{2}=\left\|F_{\bar{z}}\right\|^{2}=e^{u} / 2$ and $\left\langle F_{z}, F_{\bar{z}}\right\rangle=0$, we get readily

$$
|\nabla N|^{2}=2 \lambda_{1} e^{u}+2 \lambda_{2} e^{-u}
$$

Using (24) and (25), we have

$$
-N_{z \bar{z}}=\sqrt{\lambda_{1}} F_{z \bar{z}}+\sqrt{\lambda_{2}} e^{-u}\left(F_{\bar{z} \bar{z}}-u_{\bar{z}} F_{\bar{z}}\right)=\frac{\lambda_{1} e^{u}+\lambda_{2} e^{-u}}{2} N .
$$

This finishes the proof of the Lemma.

Hence $N$ is a harmonic map and its Hopf differential is

$$
\left\langle N_{z}, N_{z}\right\rangle=\sqrt{\lambda_{1} \lambda_{2}}
$$

Therefore, $N$ is conformal if and only if one of $\lambda_{1}, \lambda_{2}$ is zero. In this case equation (17) is just the Liouville equation.

Now we want to consider the holomorphic and anti-holomorphic part of the energy density $|\nabla N|^{2}$. The standard complex structure of $\mathbb{S}^{2}$ is given by

$$
J: T_{N} \mathbb{S}^{2} \rightarrow T_{N} \mathbb{S}^{2}, \quad J X=N \wedge X,
$$

if we consider $N \in \mathbb{S}^{2} \subset \mathbb{R}^{3}$ as a point in $\mathbb{R}^{3}$. In our expression of $\mathbb{R}^{3}$, the standard complex structure is, in terms of matrices,

$$
J: T_{N} \mathbb{S}^{2} \rightarrow T_{N} \mathbb{S}^{2}, \quad J X=\frac{1}{2}[N, X],
$$

the Lie bracket. The $\partial$-energy density and $\bar{\partial}$-energy density are

$$
e_{\partial}(N)=\frac{1}{4}\left|\frac{1}{2}\left[N, N_{x}\right]+N_{y}\right|^{2}, \quad e_{\bar{\partial}}(N)=\frac{1}{4}\left|\frac{1}{2}\left[N, N_{x}\right]-N_{y}\right|^{2}
$$

Lemma 4.3 We have

$$
e_{\partial}(N)=\lambda_{1} e^{u} \text { and } e_{\bar{\partial}}(N)=\lambda_{2} e^{-u} .
$$

Proof We can check that $\left[N, F_{z}\right]=2 i F_{z}$ and $\left[N, F_{\bar{z}}\right]=-2 i F_{\bar{z}}$ and then we use the expansion of $N_{z}$ and $N_{\bar{z}}$ by $F_{z}$ and $F_{\bar{z}}$. 
Theorem 4.4 Let $\left\{v_{n}\right\}$ be a sequence of solutions of

$$
-\Delta v_{n}=2 \lambda_{1, n} e^{v_{n}}-2 \lambda_{2, n} e^{-v_{n}} \quad \text { in } B_{r}(p) \subset \Omega .
$$

Assume that $p$ is the unique blow-up point in $B_{r}(p)$ and set

$$
m_{1}(p)=\lim _{r \rightarrow 0} \lim _{n \rightarrow \infty} \int_{B_{r}(p)} 2 \lambda_{1, n} e^{v_{n}} d x \text { and } m_{2}(p)=\lim _{r \rightarrow 0} \lim _{n \rightarrow \infty} \int_{B_{r}(p)} 2 \lambda_{2, n} e^{-v_{n}} d x
$$

Then

$$
m_{1}(p) \in 8 \pi \mathbb{N} \text { and } m_{2}(p) \in 8 \pi \mathbb{N} \text {. }
$$

Proof For each $v_{n}$, we find a unique harmonic map $N_{n}: B_{r}(p) \rightarrow \mathbb{S}^{2}$ as above with the normalization (22). In view of (26) the energy density of $N_{n}$ is

$$
e\left(N_{n}\right)=\lambda_{1, n} e^{v_{n}}+\lambda_{2, n} e^{-v_{n}} .
$$

By the assumption that $p=0$ is the only blow-up point, the sequence of harmonic maps $N_{n}$ blows up only at 0 . Now the result in [15] and [26] (see also [11]) and [20]) tells us that there are harmonic spheres $u_{j}: \mathbb{S}^{2} \rightarrow \mathbb{S}^{2}(i=\{1, \cdots, k\})$ such that (up to subsequence)

$$
\lim _{r \rightarrow 0} \lim _{n \rightarrow \infty} \int_{B_{r}(p)} e\left(N_{n}\right) d x=\sum_{j=1}^{k} E\left(u_{j}\right)
$$

It is well-known that any harmonic sphere from $\mathbb{S}^{2}$ to $\mathbb{S}^{2}$ is holomorphic or anti-holomorphic of degree $d$ with energy $4 \pi|d|$. The holomorphic energy and anti-holomorphic energy also satisfy a similar identity. In view of Lemma 4.3, we have

$$
m_{1}(p)=\lim _{r \rightarrow 0} \lim _{n \rightarrow \infty} \int_{B_{r}(p)} 2 \lambda_{1, n} e^{-v_{n}} d x=\lim _{r \rightarrow 0} \lim _{n \rightarrow \infty} 2 \int_{B_{r}(p)} e_{\partial}\left(N_{n}\right) d x \in 8 \pi \mathbb{N} .
$$

Similarly $m_{2}(p) \in 8 \pi \mathbb{N}$. This finishes the proof.

Theorem 1.1 and Corollary 1.2 follow from Theorem 4.4.

Remark 4.5 Theorem 4.4, and hence Theorem 1.1 and Corollary 1.2 hold for

$$
-\Delta v_{n}=2 \lambda_{1, n} V e^{v_{n}}-2 \lambda_{2, n} V e^{-v_{n}} \quad \text { in } B_{r}(p) \subset \Omega
$$

with a positive $C^{2}$ function $V$. In this case

$$
m_{1}(p)=\lim _{r \rightarrow 0} \lim _{n \rightarrow \infty} \int_{B_{r}(p)} 2 \lambda_{1, n} V e^{v_{n}} d x, \quad m_{2}(p)=\lim _{r \rightarrow 0} \lim _{n \rightarrow \infty} \int_{B_{r}(p)} 2 \lambda_{2, n} V e^{-v_{n}} d x .
$$

This is because all the analysis in Sect. 3 holds also on a surface $\Sigma$. Therefore, the argument utilizing harmonic maps is completely the same, and we can transfer equation (29) to (28) with a new metric $V\left(d x^{2}+d y^{2}\right)$. 


\section{Existence result}

As an application, we give an existence result for the equation (15). The functional associated to $(15)$ is

$$
J_{\lambda_{1}, \lambda_{2}}(u)=\frac{1}{2} \int_{\Omega}|\nabla v|^{2} d x-\lambda_{1} \log \left(\int_{\Omega} e^{v} d x\right)-\lambda_{2} \log \left(\int_{\Omega} e^{-v} d x\right) .
$$

It was showed in [25] and [33] that if $\lambda_{1} \leq 8 \pi$ and $\lambda_{2} \leq 8 \pi$ then there exists a positive constant $C$ such that

$$
J_{\lambda_{1}, \lambda_{2}}(u) \geq-C, \quad \text { for } u \in H_{0}^{1}(\Omega) .
$$

Inequality (30) is a generalization of the Moser-Trudinger inequality.

When $\lambda_{1}<8 \pi$ and $\lambda_{2}<8 \pi$, the existence of a solution of (15) was studied in [32] and [25]. For other existence results, see [42] and [29]. With the help of Theorem 1.1, we can consider existence of (30) for $\left(\lambda_{1}, \lambda_{2}\right) \in(8 \pi, 16 \pi) \times(0,8 \pi)$ or $\left(\lambda_{1}, \lambda_{2}\right) \in(0,8 \pi) \times(8 \pi, 16 \pi)$. Now we assume that $\Omega$ is a non simply-connected domain as in [10] for the Liouville equation.

Theorem 5.1 Let $\Omega$ be a non simply-connected domain in $\mathbb{R}^{2}$. If $\lambda_{1} \in(8 \pi, 16 \pi)$ and $\lambda_{2} \in$ $(0,8 \pi)$, then equation (15) admits a solution.

Proof The argument follows from a trick given by Struwe [37] and the blow-up analysis presented above. Since the method now becomes rather well-known (see for instance $[10,38]$ ), here we just give a sketch.

Step 1 We first define the center of mass of a function $v \in H_{0}^{1}(\Omega)$ by

$$
m_{c}(v)=\left(\int_{\Omega} e^{v} d x\right)^{-1} \int_{\Omega} x e^{v} d x
$$

Assume for simplicity that $\partial \Omega=\Gamma_{+} \cup \Gamma_{-}$has only two disjoint components. Define a family of functions

$$
\gamma: \mathbb{R} \rightarrow H_{0}^{1}(\Omega) \times H_{0}^{1}(\Omega)
$$

satisfying

$$
J_{\lambda_{1}, \lambda_{2}}(\gamma(t)) \rightarrow-\infty \quad \text { as } t \rightarrow-\infty
$$

and

$$
m_{c}(\gamma) \rightarrow \Gamma_{ \pm} \quad \text { as } t \rightarrow \pm \infty
$$

The existence of such a family is guaranteed by $\lambda_{1}>8 \pi$. Define a minimax value

$$
\alpha:=\inf _{\Gamma \in \mathcal{X}} \sup _{t \in \mathbb{R}} J_{\lambda_{1}, \lambda_{2}}(\Gamma(t)),
$$

where $\mathcal{X}$ is the set of all such families $\gamma$.

Step 2 The minimax value $\alpha>-\infty$.

Inequalty (30) can be improved under a condition introduced by Aubin [2]. 
Lemma 5.2 Let $\Omega_{1}$ and $\Omega_{2}$ be two subsets of $\bar{\Omega}$ satisfying dist $\left(\Omega_{1}, \Omega_{2}\right) \geq \delta_{0}>0$ and $\delta \in(0,1 / 2)$. For any $\epsilon>0$, there exists a constant $c=c\left(\epsilon, \delta_{0}, \delta\right)>0$ such that

$$
J_{(16 \pi-\epsilon, 8 \pi-\epsilon)}(u) \geq-c
$$

holds for all $u \in H_{0}^{1}(\Omega)$ satisfying

$$
\int_{\Omega_{1}} e^{u} d x \geq \delta \int_{\Omega} e^{u} d x \text { and } \int_{\Omega_{2}} e^{u} d x \geq \delta \int_{\Omega} e^{u} d x .
$$

Let $\Gamma_{0} \subset \Omega$ be a closed curve enclosing the inner boundary of $\Omega$. Each curve $\gamma$ starting from $\Gamma_{-}$and ending at $\Gamma_{+}$intersects with $\Gamma_{0}$. By (35), for $\left(\lambda_{1}, \lambda_{2}\right) \in(8 \pi, 16 \pi) \times(0,8 \pi)$ we can show that

$$
J_{\lambda_{1}, \lambda_{2}}(u)>-c,
$$

for any $u \in H_{0}^{1}(\Omega)$ with center of mass $m_{c}(u) \in \Gamma_{0}$. See the argument in [10]. Hence $\alpha>-\infty$.

Step 3 Now fix $\lambda_{2}$ and apply the trick of Struwe to obtain a dense subset $\Lambda$ of $(8 \pi, 16 \pi)$ such that for any pair $\left(\lambda, \lambda_{2}\right)$ with $\lambda \in \Lambda, \alpha$ is achieved by a function $v$. It is clear that $v$ is a solution of equation (15) with the pair $\left(\lambda, \lambda_{2}\right)$. This is an important step. See $[10,37]$ and [38].

Step 4 Now for any $\lambda_{1} \in(8 \pi, 16 \pi)$, there is a sequence $\lambda^{k} \in \Lambda$ with $\lambda^{k} \rightarrow \lambda_{1}$ as $k \rightarrow \infty$, since $\Lambda$ is dense. Applying Step 3, we have a solution $v_{k}$ of $(15)$ for $\left(\lambda^{k}, \lambda_{2}\right)$. Now we use the blow-up analysis established above to show that $v_{k}$ converges to $v$, which will be a solution of (15) for $\left(\lambda_{1}, \lambda_{2}\right)$. Assume by contradiction that $v_{k}$ does not converge. As discussed above, there might be two types of blow-up. We first exclude that such two types of blow-up occur at the same point $p$. Set

$$
m_{1}(p)=\lim _{r \rightarrow 0} \lim _{n \rightarrow \infty} \lambda^{k}\left(\int_{\Omega} e^{v_{k}} d x\right)^{-1} \int_{B_{r}(p)} e^{v_{k}} d x
$$

and

$$
m_{2}(p)=\lim _{r \rightarrow 0} \lim _{n \rightarrow \infty} \lambda_{2}\left(\int_{\Omega} e^{-v_{k}} d x\right)^{-1} \int_{B_{r}(p)} e^{-v_{k}} d x .
$$

By Corollary 1.2 we have

$$
\left(m_{1}(p), m_{2}(p)\right)=8 \pi\left(\frac{\ell(\ell-1)}{2}, \frac{\ell(\ell+1)}{2}\right) \text { or } 8 \pi\left(\frac{\ell(\ell+1)}{2}, \frac{\ell(\ell-1)}{2}\right) .
$$

It is trivial to see that $m_{1}(p)<16 \pi$ and $m_{2}(p)<8 \pi$. Therefore $\ell \leq 1$. Hence one of $m_{i}(p)$ must be zero, which means that the two types of blow-up cannot occur at the same point.

Since the residual term $r_{i}=0$ if $S_{i} \backslash\left(S_{1} \cap S_{2}\right) \neq \emptyset$ (see for instance [25]), we get

$$
\lim _{k \rightarrow \infty} \lambda_{k}=\sum_{p} m_{1}(p) \quad \text { or } \quad \lambda_{2}=\sum_{p} m_{2}(p),
$$

if blow-up happens. Here the summation is taken over the set of all blow-up points. Thus, $\lambda_{1}=8 \pi n_{1}$ or $\lambda_{2}=8 \pi n_{2}$ for some integers $n_{i}$. This is a contradiction. Therefore $v_{k}$ converges to $v$ which is a solution of (15) for $\left(\lambda_{1}, \lambda_{2}\right)$. 
Remark 5.3 Formula (36) might not be true (see Proposition 3.4), if there are points at which both of these two types of blow-up occur.

Remark 5.4 Theorem 5.1 is also true for (29).

Acknowledgements The work was carried out while the fourth author was visiting the Max Planck Institute for Mathematics in the Sciences. She would like to thank the institute for its hospitality and the good working conditions.

\section{References}

1. Abresch, U.: Constant mean curvature tori in terms of elliptic functions. J. Reine Angew. Math. 374, 169192 (1987)

2. Aubin, T.: Some nonlinear problems in Riemannian geometry, Springer Monographs in Mathematics, xviii+395 pp. Springer, Berlin (1998)

3. Bobenko, A.I.: All constant mean curvature tori in $\mathbb{R}^{3}, S^{3}, \mathbb{H}^{3}$ in terms of theta-functions. Math. Ann. 290, 209-245 (1991)

4. Bobenko, A.I.: Surfaces in terms of 2 by 2 matrices. Old and new integrable cases. Harmonic maps and integrable systems, pp. 83-127, Aspects Math. E23, Vieweg, Braunschweig (1994)

5. Brezis, H., Coron, J.-M.: Multiple solutions of H-systems and Rellich's conjecture. Commun. Pure Appl. Math. 37, 149-187 (1984)

6. Brezis, H., Coron, J.-M.: Large solutions of harmonic maps in two dimensions. Commun. Math. Phys. 92, 203-215 (1983)

7. Brezis, H., Merle, F.: Uniform estimates and blow-up behavior for solutions of $-\Delta u=V(x) e^{u}$ in two dimensions. Commun. Partial Diff. Equ. 16, 1223-1253 (1991)

8. Chen, X.X.: Remarks on the existence of branch bubbles on the blowup analysis of equation $-\Delta u=e^{2 u}$ in dimension two. Commun. Anal. Geom. 7, 295-302 (1999)

9. Chorin, A.J.: Vorticity and Turbulence. Springer, New York (1994)

10. Ding, W., Jost, J., Li, J., Wang, G.: Existence results for mean field equations. Ann. Inst. H. Poincaré Anal. Non Linéaire 16, 653-666 (1999)

11. Ding, W.Y., Tian, G.: Energy identity for a class of approximate harmonic maps from surfaces. Commun. Anal. Geom. 3, 543-554 (1995)

12. Hélein, F.: Régularité des applications faiblement harmoniques entre une surface et une variété riemannienne. C. R. Acad. Sci. Paris Sér. I 312, 591-596 (1991)

13. Hopf, H.: Lectures on differential geometry in the large. Stanford Lecture Notes 1955; reprinted in Lecture Notes in Math. 1000, Springer, Heidelberg (1984)

14. Jost, J.: The Dirichlet problem for harmonic maps from a surface with boundary onto a 2-sphere with non-constant boundary values. J. Diff. Geom. 19, 393-401 (1984)

15. Jost, J.: Two-dimensional geometric variational problems. Pure and Applied Mathematics (New York), Wiley, Chichester (1991)

16. Jost, J., Wang, G.: Analytic aspects of the Toda system. I. A Moser-Trudinger inequality I. A MoserTrudinger inequality. Commun. Pure Appl. Math. 54, 1289-1319 (2001)

17. Jost, J., Lin, C.S., Wang, G.: Analytic aspects of the Toda system. II. Bubbling behavior and existence of solutions. Commun. Pure Appl. Math. 59, 526-558 (2006)

18. Joyce, G., Montgomery, D.: Negative temperature states for the two-dimensional guiding-centre plasma. J. Plasma Phys. 10, 107-121 (1973)

19. Li, Y.Y., Shafrir, I.: Blow-up analysis for solutions of $-\Delta u=V e^{u}$ in dimension two. Indiana Univ. Math. J. 43, 1255-1270 (1994)

20. Lin, F.H., Wang, C.Y.: Energy identity of harmonic map flows from surfaces at finite singular time. Calc. Var. Partial Differ. Equ. 6, 369-380 (1998)

21. Lions, P.-L.: On Euler Equations and Statistical Physics. Scuola Normale Superiore, Pisa (1997)

22. Lucia, M., Nolasco, M.: SU(3) Chern-Simons vortex theory and Toda Systems. J. Differ. Equ. 184, 443474 (2002)

23. Marchioro, C., Pulvirenti, M.: Mathematical Theory of Incompressible Nonviscous Fluids. Springer, New York (1994)

24. Newton, P.K.: The N-Vortex Problem: Analytical Techniques. Springer, New York (2001)

25. Ohtsuka, H., Suzuki, T.: Mean field equation for the equilibrium turbulence and a related functional inequality. Adv. Differ. Equ. 11, 281-304 (2006) 
26. Parker, T.H.: Bubble tree convergence for harmonic maps. J. Differ. Geom. 44, 595-633 (1996)

27. Pinkall, U., Sterling, I.: On the classification of constant mean curvature tori. Ann. Math. 130, 407451 (1989)

28. Pointin, Y.B., Lundgren, T.S.: Statistical mechanics of two dimensional vortices in a bounded container. Phys. Fluids 19, 1459-1470 (1976)

29. Ricciardi, T.: Mountain pass solutions for a mean field equation from two-dimensional turbulence. ArXiv: math. AP/0612123 (2006)

30. Rivière, T.: Conservation laws for conformal invariant variational problems. ArXiv math. AP/0603380 (2006)

31. Sacks, J., Uhlenbeck, K.: The existence of minimal immersions of 2-spheres. Ann. Math. 113, 1-24 (1981)

32. Sawada, K., Suzuki, T., Takahashi, F.: Mean field equation for equilibrium vortices with neutral orientation. Nonlinear Anal. 66(2), 509-526 (2007)

33. Shafrir, I., Wolansky, G.: Moser-Trudinger and logarithmic HLS inequalities for systems. J. Eur. Math. Soc. 7, 413-448 (2005)

34. Spruck, J.: The elliptic sinh Gordon equation and the construction of toroidal soap bubbles. Calculus of variations and partial differential equations (Trento, 1986), pp. 275-301, Lecture Notes in Math. vol. 1340, Springer, Berlin (1988)

35. Steffen, K.: On the nonuniqueness of surfaces with prescribed constant mean curvature spanning a given contour. Arch. Rat. Mech. Anal. 94, 101-122 (1986)

36. Struwe, M.: Nonuniqueness in the Plateau problem for surfaces of constant mean curvature. Arch. Rat. Mech. Anal. 93, 135-157 (1986)

37. Struwe, M.: Plateau's problem and the calculus of variations. Mathematical Notes 35, Princeton University Press, Princeton (1988)

38. Struwe, M., Tarantello, G.: On multivortex solutions in Chern-Simons gauge theory. Boll. Unione M at. Ital. Sez. B Artic. Ric. Mat. 1(8), 109-121 (1998)

39. Wente, H.: An existence theorem for surfaces of constant mean curvature. J. Math. Anal. Appl. 26, 318344 (1969)

40. Wente, H.: Large solutions of the volume constrained Plateau problem. Arch. Rat. Mech. Anal. 75, 59$77(1980)$

41. Wente, H.: Counterexample to a conjecture of H. Hopf. Pacific J. Math. 121, 193-243 (1986)

42. Zhou, C.Q.: Existence of solution for mean field equation for the equilibrium turbulence. Preprint (1986) 